OPEN ACCESS

Edited by:

Anna Kramvis,

University of the Witwatersrand,

South Africa

Reviewed by: Barbara Rehermann,

National Institutes of Health (NIH),

United States

Masaya Sugiyama

National Center for Global Health

and Medicine, Japan

*Correspondence:

Hui Zhuang

zhuangbmu@126.com

Tong Li

tongli08@vip.sina.com

Specialty section:

This article was submitted to

Virology,

a section of the journal

Frontiers in Microbiology

Received: 24 January 2019

Accepted: 29 May 2019

Published: 12 June 2019

Citation:

Xiang K, Xiao Y, Li Y, He L, Wang L, Zhuang H and Li T (2019)

The Effect of the Hepatitis B Virus Surface Protein Truncated sC69*

Mutation on Viral Infectivity

and the Host Innate Immune Response. Front. Microbiol. 10:1341.

doi: 10.3389/fmicb.2019.01341

\section{The Effect of the Hepatitis B Virus Surface Protein Truncated sC69* Mutation on Viral Infectivity and the Host Innate Immune Response}

\author{
Kuanhui Xiang, Yiwei Xiao, Yao Li, Lingyuan He, Luwei Wang, Hui Zhuang* and Tong Li*
}

Department of Microbiology and Infectious Disease Center, School of Basic Medical Sciences, Peking University Health Science Center, Beijing, China

Viruses could rapidly diversify into variants, which has long been known to facilitate viral adaption in the host. Recent studies showed that cooperation among variants and wild-type (WT) also increased viral fitness. Here, a mutant of sC69* in small hepatitis B surface protein (SHBs) that resulted in premature stop was investigated and the frequency of sC69* was $4.37 \%$ (19/435), most of which coexisted with the WT $(78.95 \%$, 15/19), indicating mixed viral populations. Functional studies showed that sC69* mutant was associated with lower viral spread, but could be rescued by coexisting with the WT. The sC69* mutant showed to attenuate host innate immune response during infection and poly (I:C) treatment such as IL29, ISG15, and RIG-I ( $p<0.05)$. The lower immune response was not caused by the lower replication of sC69* mutant. Our data provide information that sC69* coexisting with the WT might facilitate the fitness and persistence of the viral quasispecies in the host.

Keywords: HBV, truncated mutant, sC69*, viral infectivity, innate immune response

\section{BACKGROUND}

Hepatitis B surface antigen (HBsAg) is a major viral protein of hepatitis B virus (HBV) circulating in patient serum and serves as an important virological marker for the evaluation of chronic HBV infection and antiviral response. We found that 4.37\% (19/435) of the studied patients with genotype C HBV chronic infection carried the small hepatitis B surface (SHBs) sC69* mutant resulting in the truncated SHBs [about 1/3 length of wild-type (WT) SHBs]. This mutant usually existed in older people with lower HBV DNA levels. In addition, we found that the sC69* negatively impaired viral replication, infectivity and spread, which could be rescued by the WT. On the contrary, the $\mathrm{sC}^{*}{ }^{*}$ mutant inhibited the host innate immune response more potently than that of the WT. These results indicate that HBV sC69* coexisting with WT might facilitate the viral fitness and surviving from the host surveillance. 


\section{INTRODUCTION}

Despite the availability of an effective vaccine and the improvement of antiviral treatment, chronic HBV infection remains a severe public health problem. According to the global hepatitis report in 2017 from World Health Organization (WHO), estimated 257 million people worldwide are chronically infected, with an increased risk for developing liver cirrhosis and hepatocellular carcinoma (Liang T.J. et al., 2015).

Hepatitis B virus surface protein consists of three related yet different proteins, the large, middle and small hepatitis $B$ surface proteins (LHBs, MHBs, and $\mathrm{SHBs}$, respectively). The SHBs has a MHR, including the 'a' determinant from AA 124-147, which is a dominant neutralizing epitope and most neutralizing antibodies target to (Locarnini et al., 2012). Naturally occurring AA variations in this region are prevalent and affect HBsAg antigenicity, secretion, circulating HBsAg and HBV DNA levels (Alavian et al., 2013). Huang et al. (2012) reported that the AA variations in the 'a' determinant could result in lower serum HBsAg levels in patients with occult HBV infection (Wu C.C. et al., 2012).

However, HBsAg AA variations naturally exist not only within the 'a' determinant region, but also within its flanking regions (Warner and Locarnini, 2008; Alavian et al., 2013). Recently, it was shown that HBsAg truncated mutants such as sW172*, sW182*, and sW199* occurred in patients both with and without antiviral treatment (Warner and Locarnini, 2008; Pollicino et al., 2012). Apart from these findings, another truncated mutant (sC69*) was identified from CHB patients as shown in previous studies (Betz-Stablein et al., 2016; Xiang et al., 2017). Recently, the sC69* was found in genotype A CHB patients, which was reported to be associated with entecavir (ETV) and tenofovir (TDF) resistance (Shirvani-Dastgerdi et al., 2017). Interestingly, these truncated mutants could inhibit viral replication and secretion (Warner and Locarnini, 2008; Xiang et al., 2017). Our previous study showed that the $\mathrm{sC} 69^{*}$ mutant could restrict viral replication and secretion, but these defects could somehow be rescued by the WT HBs (Xiang et al., 2017). However, little is known about the influence of the sC69* mutant on viral infectivity and spread. Yan et al. (2012) reported that NTCP was a receptor for HBV infection, and NTCP overexpression in HepG2 cells was shown to confer susceptibility to HBV infection. Thus, it is possible to use HepG2-NTCP cells as an HBV infection model to study the influence of sC69* on viral infectivity and spread.

Recently, many studies have suggested that cooperative interactions among viral quasispecies (among variants and WT)

\footnotetext{
Abbreviations: AA, amino acid; AFP, alpha-fetoprotein; Alb, albumin; ALT, alanine aminotransferase; AST, aspartate aminotransferase; cccDNA, covalently closed circular DNA; CHB, chronic hepatitis B; CLIA, chemiluminescence assay; DAPI, 4',6-diamidino-2-phenylindole; DMSO, dimethyl sulfoxide; HBsAg, hepatitis B surface antigen; HBV, hepatitis B virus; HCM, hepatocyte culture medium; hESCs, human embryonic stem cells; HGF, hepatic growth factor; HLCs, induced pluripotent stem cell derived hepatocyte-like cells; ISGs, interferon stimulated genes; IU/ml, international unit per milliliter; LHBs, large hepatitis B surface protein; LMV, lamivudine; MHBs, middle hepatitis B surface protein; MHR, major hydrophilic region; NTCP, sodium taurocholate cotransporting polypeptide; qHBsAg, quantitative hepatitis B surface antigen; RT, reverse transcriptase; SHBs, small hepatitis B surface protein; WT, wild-type.
}

support viral population fitness. For example, it was reported that a population with poliovirus variants was required for pathogenesis, suggesting that cooperative interactions among the variants and WT played important roles in viral fitness and survival (Pfeiffer and Kirkegaard, 2005). More recently, the cooperative interactions were also reported in measles virus and Coxsackie virus (Shirogane et al., 2012; Borderia et al., 2015). Similarly, it was also shown that HBV WT rescued the replication and secretion of variants such as sW172*, sW182*, and sC69* (Warner and Locarnini, 2008; Pollicino et al., 2012; Xiang et al., 2017). However, the reason why HBV replication-deficient variants exist in host or the significance of their coexistence with the WT is still not clearly understood. Our hypothesis for sC69* is that it can inhibit host innate immune responses, which does not only protect itself but also the WT from host immune surveillance.

As reported before, host immune response against $\mathrm{HBV}$ infection is crucial for better understanding of the pathological processes and viral elimination to control HBV infection (Rehermann and Nascimbeni, 2005). Lucifora et al. (2014) reported that $\mathrm{APOBEC} 3 \mathrm{~A} / 3 \mathrm{~B}$ cytidine deaminases induced by interferon (IFN) could deaminate cytosines in HBV cccDNA and lead to cccDNA degradation. Shlomai et al. (2014) reported that $\mathrm{HBV}$ infection could induce the IFN stimulated genes (ISGs) response in primary hepatocytes cultured in micro-patterned cocultures of primary human hepatocytes with stromal cells. Yan et al. (2015a) showed that the IFN inducible protein, tetherin, inhibited HBV secretion. However, HBV has many strategies to escape from innate immune surveillance. For example, Liu S. et al. (2015) reported that HBsAg and hepatitis B e antigen (HBeAg) could inhibit major vault protein to negatively impair response to IFN therapy. In addition, HBV polymerase was shown to inhibit K63 ubiquitination of STING, thereby negatively impacting the innate immune response (Liu Y. et al., 2015). Interestingly, Chen et al. (2015) showed that HBV spliced variants were associated with negatively impaired response to IFN therapy. It was recently reported that cooperative interactions among two HBsAg mutants at different ratio could increase HBV replication and influence the host immune response and antibody response (Cao et al., 2014). We therefore wondered if the sC69* mutant could also inhibit innate immune responses and promote HBV persistence in the host.

To characterize how the sC69* mutant exists in the host, we sequenced the $\mathrm{SHBs}$ coding region in $\mathrm{HBV}$ genotype $\mathrm{C}$ from a cohort of $435 \mathrm{CHB}$ patients without or with antiviral therapy. We found that 19 patients harbored the sC69* mutant. We then performed in vitro experiments to study the impact of the sC69* on viral replication, infectivity and spread and innate immune responses.

\section{RESULTS}

\section{Truncated Mutation at Site 69 in S Gene Tend to Occur in Mixed Population}

Among 435 CHB patients, we identified 19 samples carrying $\mathrm{HBV}$ sC69* mutant by analyzing entire SHBs AA 
sequences. We grouped the patients into sC69 WT and sC69* groups (Table 1).

In order to know the distribution of sC69* mutant in different patient groups, we stratified patients by HBeAg status and LMV exposure and analyzed the SHBs sequences. This showed that the frequency of $\mathrm{s} 69^{*}$ mutant varied in different CHB patients, including HBeAg-positive untreated patients (3.67\%, 12/327), HBeAg-negative untreated patients $(11.90 \%$, $5 / 42)$ and LMV-treated patients $(3.03 \%, 2 / 66)$ (Table 2). Of note, the sC69* significantly existed at a higher frequency in $\mathrm{HBeAg}$-negative untreated $\mathrm{CHB}$ patients in comparison to that in HBeAg-positive ones $(p<0.05)$. In addition, we found that the $\mathrm{sC} 69^{*}$ mutant usually coexisted with WT sC69 shown by double peaks at the third nucleotide of the sC69 codon (TGT and TGA) in PCR direct sequencing electropherograms (Supplementary Figure S1A). Table 2 showed that $78.95 \%(15 / 19)$ of the $\mathrm{sC} 69^{*}$ mutations were identified as $\mathrm{sC} 69 \mathrm{C} /{ }^{*}$ quasispecies, indicating that $\mathrm{s} 69^{*}$ usually coexisted with the WT.

\section{The Negative Impact of the sC69* Mutant on Viral Infection Could Be Rescued by Co-expression of WT HBs}

We showed before that the $\mathrm{sC}^{\mathrm{C}} \mathrm{9}^{*}$ inhibits virion replication and secretion which can be rescued by co-expressing WT HBs to facilitate this mutant survival, indicating that WT surface proteins can package the sC69* mutant genome and allow the mutant to persist (Supplementary Figures S1B,C) (Xiang et al., 2017). The sC69* not only inhibits viral secretion, but also results in HBs locating around the hepatocyte nuclear (Supplementary Figure S1D).

HepG2-NTCP cell line has been established (Supplementary Figure S2), which highly supported HBV infection and could be used to study sC69* in vitro in this study (Michailidis et al., 2017).

TABLE 1 | Comparison of clinical data between patients with and without sC69* substitution.

\begin{tabular}{|c|c|c|c|}
\hline \multirow[t]{2}{*}{ Characteristics } & \multicolumn{2}{|c|}{ Patients } & \multirow[t]{2}{*}{$P$-value } \\
\hline & WT (416) & sC69* (19) & \\
\hline $\begin{array}{l}\text { Age (years), median } \\
\text { (range) }\end{array}$ & $36(13-81)$ & $46(21-68)$ & 0.004 \\
\hline Gender, male (\%) & $280(67.3 \%)$ & $13(68.4 \%)$ & NS \\
\hline LMV treatment (ratio\%) & 64 (15.4\%) & $2(10.5 \%)$ & NS \\
\hline $\begin{array}{l}\text { ALT (U/L), median } \\
\text { (range) }\end{array}$ & $92.0(24.1-1393.0)$ & $87.0(28.0-1150.0)$ & NS \\
\hline $\begin{array}{l}\text { AST (U/L), median } \\
\text { (range) }\end{array}$ & $70.0(22.2-1207.0)$ & $94.00(39.0-985.0)$ & NS \\
\hline $\begin{array}{l}\text { HBV DNA }\left(\log _{10} \mid \mathrm{IU} / \mathrm{ml}\right) \text {, } \\
\text { median (range) }\end{array}$ & $7.6(3.1-10.6)$ & $5.73(3.9-9.4)$ & 0.02 \\
\hline $\begin{array}{l}\mathrm{HBsAg}\left(\log _{10} \mathrm{IU} / \mathrm{ml}\right) \text {, } \\
\text { median (range) }\end{array}$ & $4.2(2.4-5.5)$ & $3.74(3.0-4.2)$ & NS \\
\hline HBeAg (positive\%) & 361 (86.8\%) & $14(73.7 \%)$ & NS \\
\hline
\end{tabular}

ALT, alanine aminotransferase; AST, aspartate transaminase; $H B A A g$, hepatitis $B$ surface antigen; $\mathrm{HBeAg}$, hepatitis B e antigen; NS, no significance; WT, wild-type.
TABLE 2 | The prevalence of sC69* in HBV infection.

\begin{tabular}{lcccc}
\hline Patients & $\begin{array}{c}\text { Number of } \\
\text { patients }\end{array}$ & \multicolumn{3}{c}{ Codon at sC69 } \\
\cline { 3 - 5 } & & $\begin{array}{c}\text { TGT (sC69) } \\
(\%)\end{array}$ & $\begin{array}{c}\text { TGA (sC69*) } \\
(\%)\end{array}$ & $\begin{array}{c}\text { TGT/TGA } \\
\text { (sC69C/*) (\%) }\end{array}$ \\
\hline $\begin{array}{l}\text { HBeAg-positive } \\
\text { untreated }\end{array}$ & 327 & $315(96.33)$ & $3(0.92)$ & $9(2.75)$ \\
$\begin{array}{l}\text { HBeAg-negative } \\
\text { untreated }\end{array}$ & 42 & $37(88.10)$ & $1(2.38)$ & $4(9.52)$ \\
$\begin{array}{l}\text { LMV-treated } \\
\text { Total }\end{array}$ & 66 & $64(96.97)$ & $0(0.00)$ & $2(3.03)$ \\
& 435 & $416(95.63)$ & $4(0.92)$ & $15(3.45)$
\end{tabular}

HBeAg, hepatitis B e antigen; LMV, lamivudine.

To determine whether sC69* mutant could impact virion infection and spread, we modified the experimental system by using the transwell plate, in which the transfection-produced virions were generated in the upper insert and penetrated to the bottom well containing HepG2-NTCP cells (Figure 1A). Interestingly, WT HBV infection resulted in high positive infected cells, while the $\mathrm{s} 69^{*}$ mutant showed no sign of infected cells. However, HepG2-NTCP cells infected by the virions produced by co-transfecting WT HBs expression plasmid pLMS and full viral genome encoding the $\mathrm{s} C 69^{*}$ mutant showed high percentage of hepatitis $\mathrm{B}$ core antigen $(\mathrm{HBcAg})$ staining positive cells similar to that of the WT infection, indicating that WT HBs can package the sC69* mutant genome and help it infect new cells (Figure 1B). And also, trans-complementary experiment showed similar HBV pgRNA level to WT infection in HepG2NTCP cells (Figure 1C). Taken together, the results suggested that the $\mathrm{sC}^{*} 9^{*}$ mutant could negatively impact viral infection and spread, while WT HBs could rescue $5 \mathrm{C} 69^{*}$ mutant infectivity and spread. Thus, the sC69* + pLMS and WT were used in the following experiments.

\section{The Impact of the sC69* Mutant on Innate Immune Response in HepG2-NTCP Cell Line}

HepG2-NTCP cell line was competent for innate immune response with treatment of $10 \mu \mathrm{g} / \mathrm{ml}$ poly (I:C) overnight (Supplementary Figure S3), indicating that HepG2-NTCP cells could be stimulated to produce innate immune response. To ascertain whether sC69* could affect innate immune responses, we infected HepG2-NTCP cells in transwell system and quantified mRNA levels of innate immunity related genes (Figure 2A). As shown in Figure 2B, HBV pgRNA from WT and $\mathrm{s} 69^{*}+$ pLMS increased over time. However, the pgRNA levels from WT are higher than sC69* + pLMS at day 3 of viral infection. Interestingly, many ISGs and cytokine mRNAs such as IL29, ISG15 and RIG-I $(p<0.05)$ were stimulated by WT HBV infection. However, when HepG2-NTCP cells were infected with sC69* + pLMS, most of ISGs and cytokines mRNAs were lower compared to WT at day 3 (IL29 and ISG15) and day 5 (RIG-I) $(p<0.05)$. In addition, their expression was not largely improved in $\mathrm{s} 66^{*}+$ pLMS-derived virus infection, indicating 

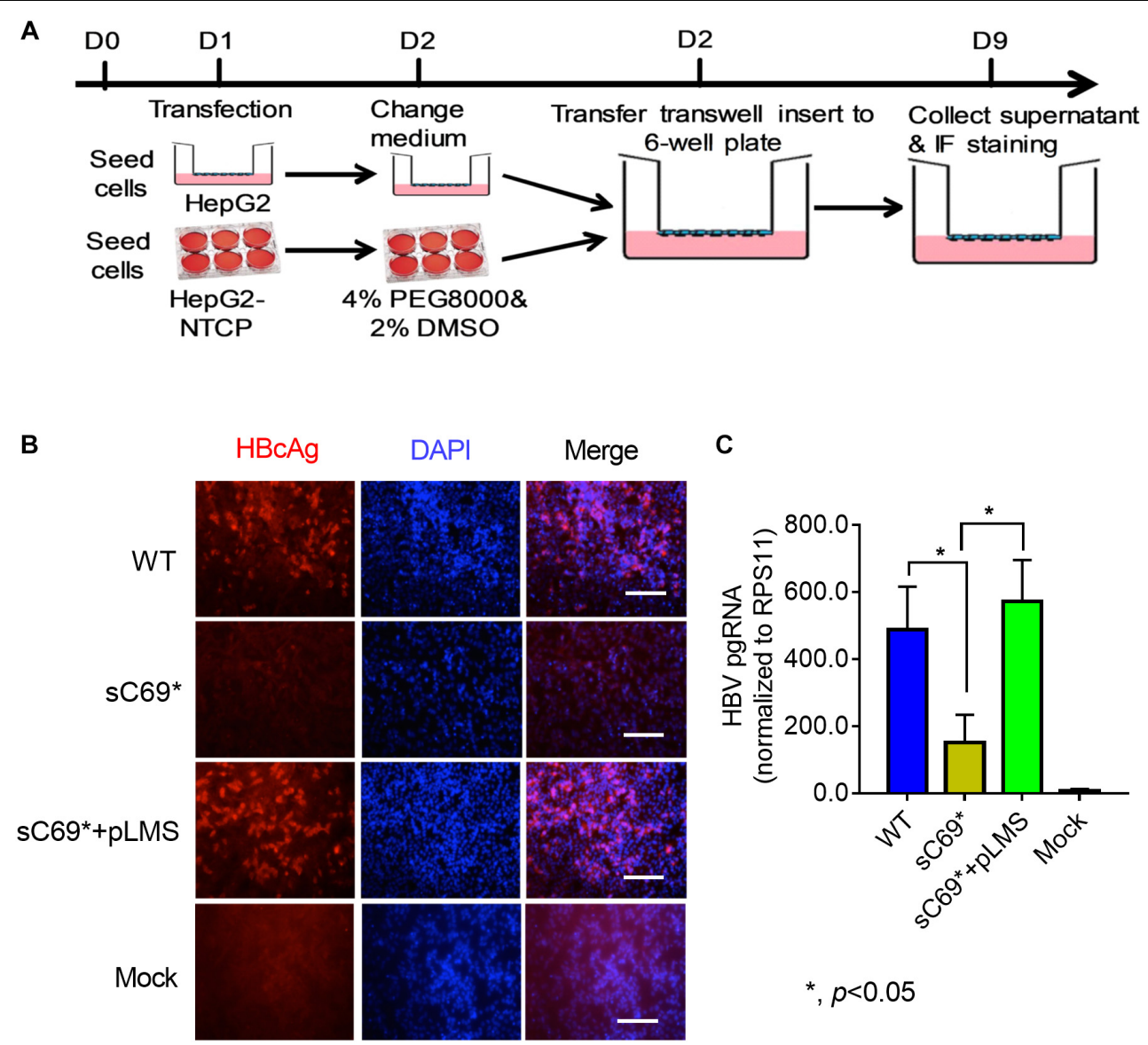

FIGURE 1 | The viral infectivity affected by sC69* substitution could be rescued when co-existence with wild-type (WT) HBsAg. (A) The schematic strategy was used to test HBV infectivity in the trans-well system. To avoid the impact of collecting and concentrating steps on viral activity, we tried to use the trans-well system to test HBV infection. HepG2 cells seeded in the insert of trans-well were transfected with HBV WT, sC69* and sC69* + pLMS, respectively. The next day, the inserts were transferred to wells with HepG2-NTCP cells. Seven days post-infection, HBV related marker were detected. (B) Immunofluorescent microscopy of HBcAg in the WT, sC69* and sC69* + pLMS infection, scale bars: $100 \mu \mathrm{m}$. (C) RT-qPCR detection of HBV pgRNA at different groups. Data from three biological replicates are shown as means \pm s.d. Statistical analysis was performed by a Student's $t$-test * $p<0.05$.

that sC69* could inhibit innate immune responses to escape innate immune surveillance.

As shown before, the sC69* mutant could inhibit viral replication ability. To exclude the viral replication influence on the induction of innate immune response, we did RT mutation of $\mathrm{HBV}$ WT and sC69* from YMDD to YMHD (Supplementary Figure S4A). This mutation could inhibit viral replication. The expression of viral proteins and RNAs from the transfected plasmids would show similar levels for WT and sC69*. Therefore, we could exclude the possibility that the less induction of innate immune response were caused by the less replication of sC69* mutant. We transfected WTYMHD and sC69*-YMHD into HepG2 cells and detect HBV pgRNA and ISGs expression at days $0.5,1,2,3,5$, and 7 post-transfection. As shown in Supplementary Figure S4B, the expression of HBV pgRNA were almost the same between WTYMHD and sC69*-YMHD. However, some of the ISGs were still significantly higher 3 days post-transfection in WT-YMHD than those in sC69*-YMHD, such as IL29, ISG15, RIG-I and viperin $(p<0.05)$. The expression of TNF $\alpha$, IL1 $\beta$, and IL32 was the same during the days post-transfection between WTYMHD and sC69*-YMHD. These results indicate that the less induction of innate immune response was not caused by less viral replication. Maybe the sC69* mutant itself antagonizes the innate immune response.

In order to study if WT and sC69* induce effective IFNs expression, we transferred the supernatant from WT and sC69* + pLMS infected HepG2-NTCP cells to new cells without infection and harvested the cellls $6,12,24$, and $48 \mathrm{~h}$ post-transferring (Supplementary Figure S5A). We detected pgRNA and HBV total RNA levels from the infected cells. The data (Supplementary Figure S5B) showed that WT had higher pgRNA and total RNA expression than sC69* + pLMS, which meant that HepG2-NTCP cells supported well WT and lower sC69* mutant infection. However, when transferring the supernatants to new cells, we couldn't find any ISGs expression, while the cells were induced with some ISGs expression under poly (I:C) treatment (Supplementary Figure S5C). 


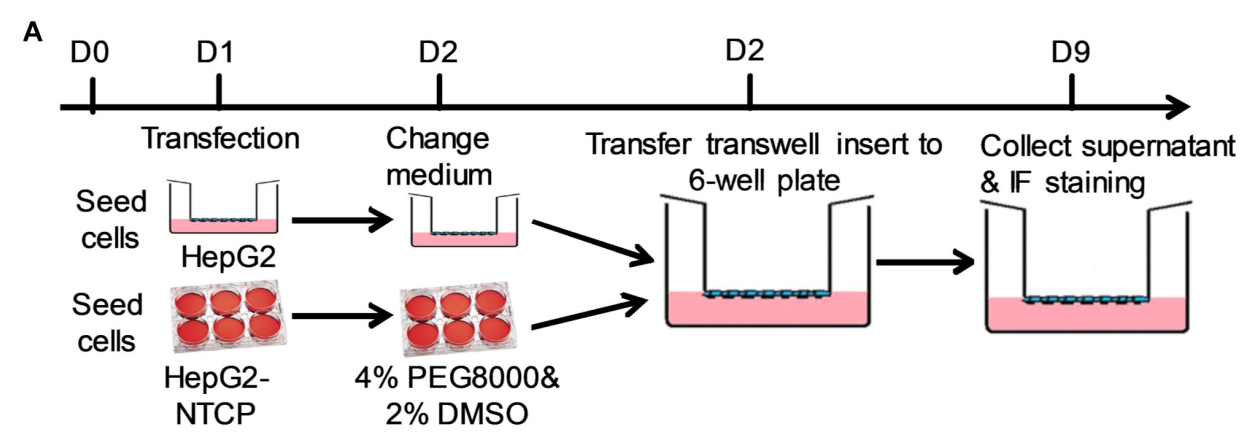

B

HBV pgRNA

TNF $\alpha$
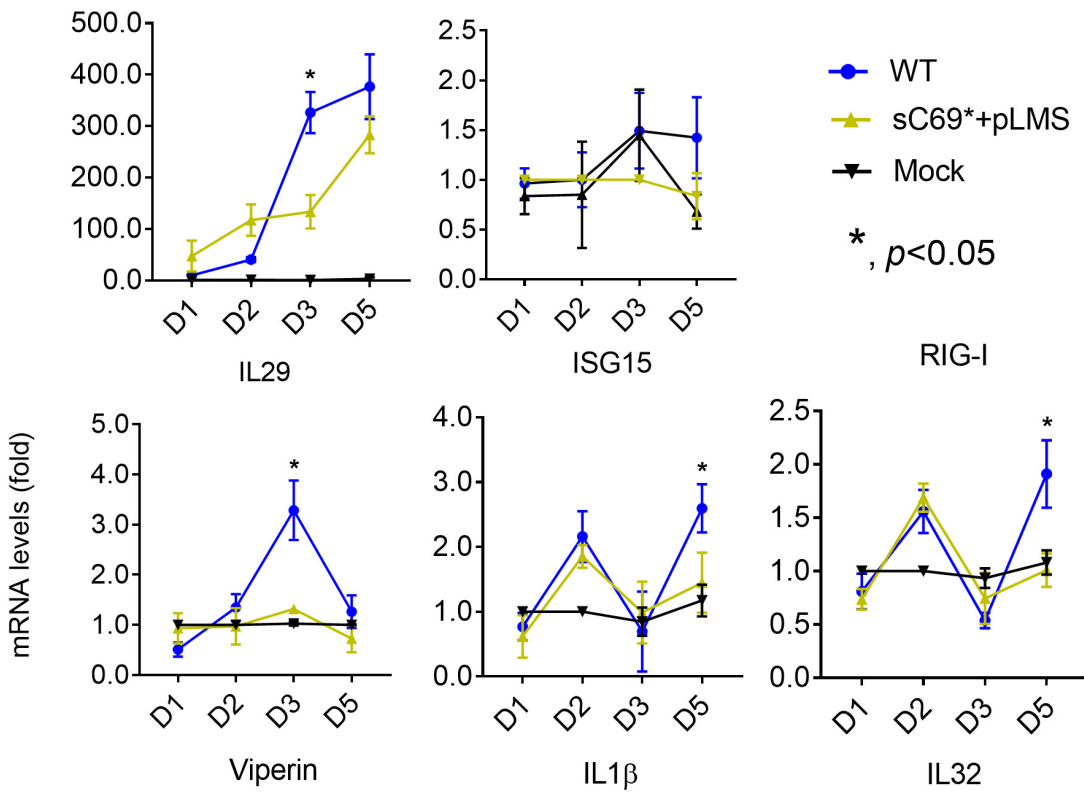

RIG-I
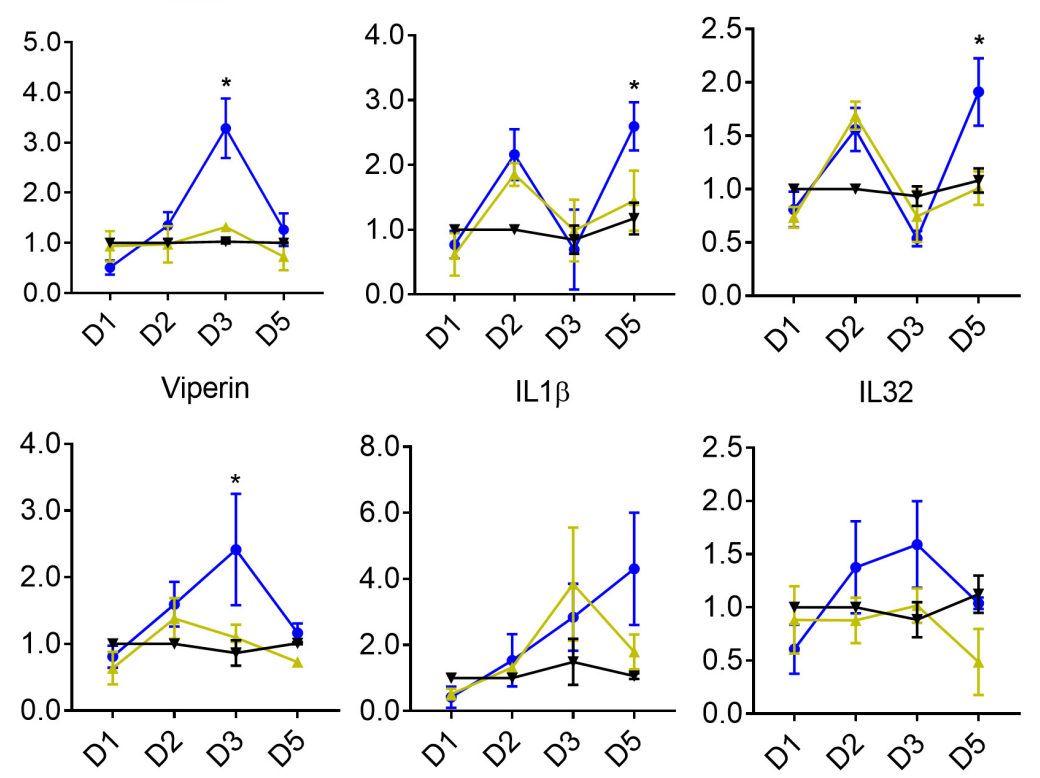

Days post-infection

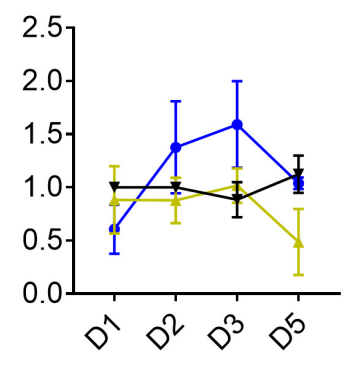

FIGURE 2 | The sC69* mutant inhibits host innate immune response compared to HBV WT in HepG2-NTCP cells. (A) HepG2 cells seeded in the insert of trans-well were transfected with HBV WT and sC69* + pLMS, respectively. The next day, the inserts were transferred to wells with HepG2-NTCP cells. The cells were harvested at days 1, 2, 3, and 5 post-infection. (B) HBV pgRNA and innate immunity related mRNAs (TNF $\alpha$, IL29, ISG15, RIG-I, viperin, IL1 $\beta$, and IL32) were detected after the infection of HBV virions from transfection system. Data from three biological replicates are shown as means \pm s.d. Statistical analysis was performed by a Student's $t$-test * $p<0.05$.

\section{The sC69* Mutant Attenuates the Innate Immune Response During Poly (I:C) Treatment}

Given the very low induction of ISGs expression by sC69* + pLMS in HepG2-NTCP cells, we explored if sC69* mutant could attenuate poly (I:C) mediated immune response by monitoring the activation of ISGs expression. We stimulated HepG2-NTCP cells with $10 \mu \mathrm{g} / \mathrm{ml}$ poly (I:C) during HBV WT and sC69* + pLMS infection (Figure 3A). The levels of HBV pgRNA and total RNA of sC69* + pLMS was similar to those of WT (Figure 3B). Consistent to Figure 2, ISGs expression in the sC69* + pLMS infected cells was significantly lower compared 
A Infect HBV

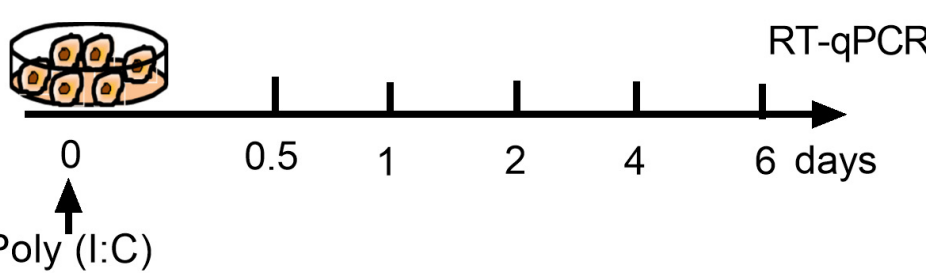

B

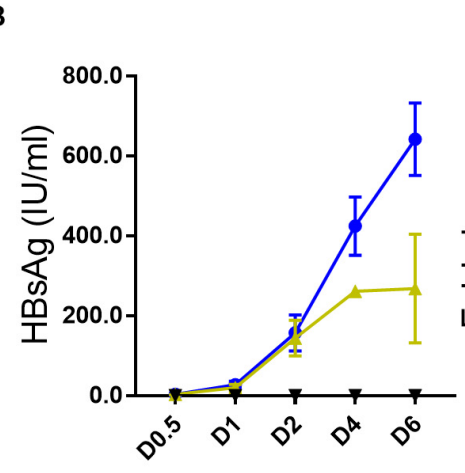

Days post-infection

C

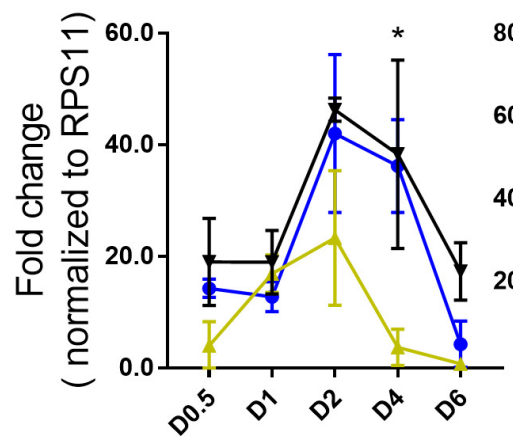

Days post-infection

TNF $\alpha$

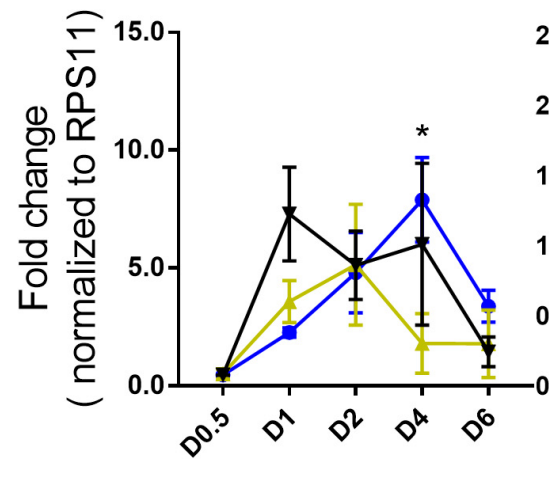

Days post-infection

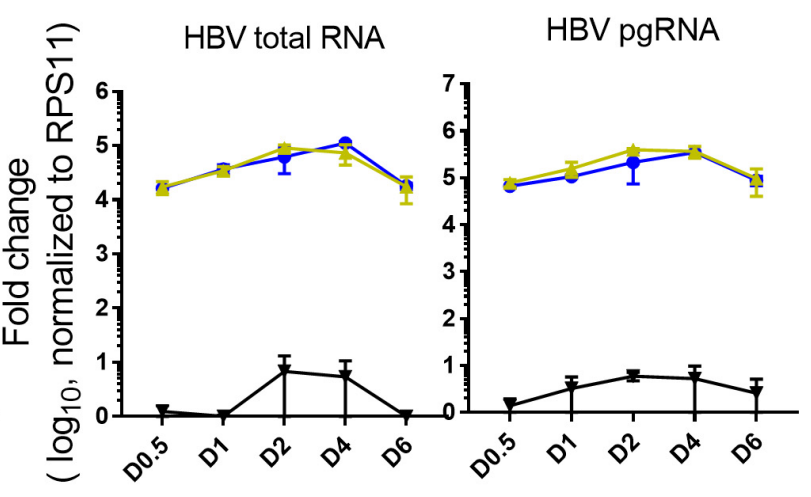

Days post-infection Days post-infection

IL29

RIG-I

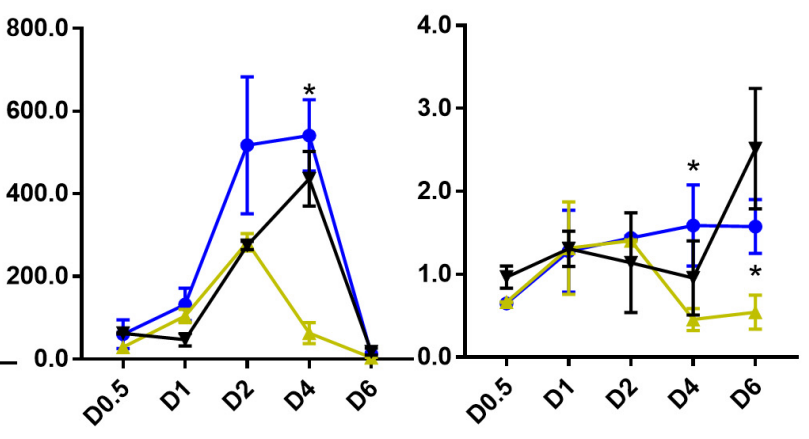

Days post-infection

Days post-infection

IL32

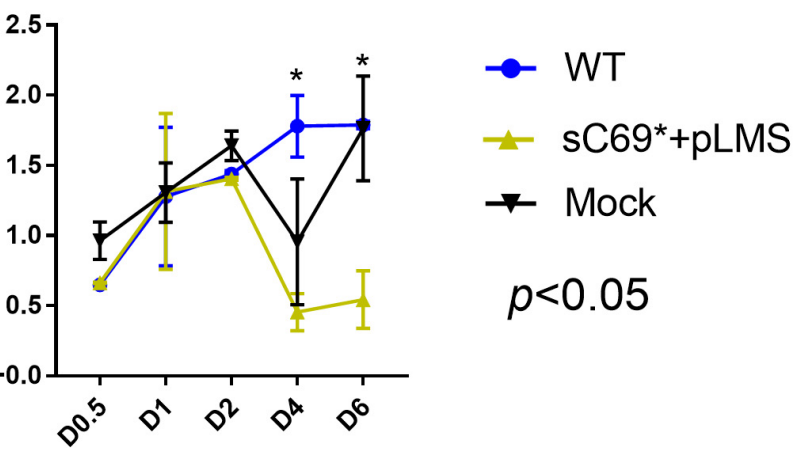

Days post-infection

FIGURE 3 | The sC69* mutant attenuates the poly (l:C) inducing host immune response in HepG2-NTCP cells. (A) Schematic workflow for detecting ISGs expression during HBV WT and sC69* + pLMS infection with poly (I:C) treatment in HepG2-NTCP cells. Briefly, we collected virions from HBV WT and sC69* + pLMS transfections that were used to, infect HepG2-NTCP cells with $10 \mu \mathrm{g} / \mathrm{ml}$ poly (I:C) during the infection and measured the viral and ISGs related 
FIGURE 3 | Continued

marker 0.5, 1, 2, 4, and 6 days post-infection. (B) Representative expression of HBsAg, pgRNA, and HBV total RNA levels during the infection. (C) Expression of IL28 $\beta$, IL29, RIG-I, TNF $\alpha$, and IL32 mRNA levels were presented during the time course of viral infection. Data from three biological replicates are shown as

means \pm s.d. Statistical analysis was performed by a Student's $t$-test * $p<0.05$.

to WT and poly (I:C) treated only cells (mock) (Figure 3C), suggesting that sCS69* could attenuate poly (I:C) stimulated ISGs expression.

To examine whether sC69* attenuates ISGs expression at early stage or late stage (IFNs mediated signaling) of ISGs induction, we stimulated WT and sC69* + pLMS infected HepG2-NTCP cells with $500 \mathrm{U} / \mathrm{ml}$ IFN $\beta$ during the infection and measured the expression of pgRNA, HBV total RNA and ISGs levels by RT-qPCR (Figure 4A). Consistent to previous study, expression of pgRNA and HBV total RNA in IFN $\beta$ untreated group were significantly higher than those with IFN $\beta$ treatment both in WT and sC69* + pLMS (Figure 4B). However, there were no difference of ISGs expression between WT and sC69* + pLMS (Figure 4C), indicating that sC69* attenuates of ISGs expression at early stage of innate immune response.

\section{In vitro HLCs Are Permissive for HBV Infection}

To further confirm the novel findings obtained in HepG2NTCP, we used HLCs derived from hESCs as a model to study HBV WT and mutant infection and related innate immune responses. Firstly, we investigated whether hESCderived hepatocytes were permissive to $\mathrm{HBV}$ infection. The special markers for hESCs differentiation to HLCs were showed (Supplementary Figure S6). We used 500 geq/cell of HBV virions from HepDE19 cells to infect HLCs for 7 days. As shown in Supplementary Figure S7A, the HBV receptor of NTCP was highly expressed in these HLCs. HBcAg staining (Supplementary Figure S7B) and HBV pgRNA (Supplementary Figure S7C) from the cells, as well as HBsAg (Supplementary Figure S7D), HBeAg (Supplementary Figure S7E) and HBV DNA from the supernatant (Supplementary Figure S7F) were positive after HBV infection compared to negative control, indicating differentiated hepatocytes derived from hESCs were permissive for $\mathrm{HBV}$ infection.

\section{The Impact of the sC69* Mutant on Innate Immune Response in the HLCs Model}

To study whether the sC69* mutant could affect innate immune responses; we carried out transwell-based infection experiment. Firstly, we transfected the WT and sC69* + pLMS into HepG2 cells seeded in the insert of the transwell. The next day we transferred the insert into plates seeded with HLCs and harvested the cells at different time points (Figure 5A). Interestingly, consistent with HBV infection in HepG2-NTCP system, the sC69* + pLMS showed significantly lower innate immune responses than WT regarding the expression of IL29, ISG15, and RIG-I mRNA levels 4 days post-infection and TNF $\alpha 7$ days post infection $(p<0.05)$, while the other ISGs expression seemed to show overall lower trend in sC69* + pLMS than that of WT but without statistical difference (Figure 5B), indicating that sC69* could attenuate the expression of some ISGs.

In order to study if the truncated protein attenuates the innate immune response, we used the plasmid of pLMS to study the impact of the truncated protein on innate immune response. We found that it has IFNs induction, with similar trend of ISGs expression to the replicon based results (Figure 6), indicating that the truncated protein could have an effect on the innate immune response.

\section{DISCUSSION}

In this study, we reported that the sC69* mutant was highly prevalent in the $435 \mathrm{CHB}$ patients with and without antiviral treatment. Patients with the sC69* mutant were significantly older than those with WT infection. In addition, HBV DNA levels in patients with the $\mathrm{sC} 69^{*}$ mutant were significantly lower than those where sC69* was not detected. The sC69* mutant exists mostly in $\mathrm{HBeAg-negative} \mathrm{CHB}$ patients and usually coexisted with WT as quasispecies. In vitro studies showed that the $\mathrm{sC}^{2} 9^{*}$ mutant could inhibit viral infection and spread, but could be rescued by WT surface protein. It was reported that HBsAg could be transcribed from HBV sequences that are integrated into the host genome rather than from replicating virus (Wooddell et al., 2017). In our study, we could detect HBV pgRNA levels, which supports the possible origin of sC69* from cccDNA. Therefore, our in vitro models should be able to resemble that occurring in vivo at least to some extent.

The sC69* mutant was reported in several published papers including our previous reports (Ding et al., 2014; Xiang et al., 2017). It not only exists in treatment-naïve patients, but also exists in LMV, ETV, and TDF treated patients (Xiang et al., 2017). However, in a recent study, Shirvani-Dastgerdi et al. (2017) reported that sC69* in genotype A HBV had a higher viral replication. The reason for this difference may be due to different aspects, such as genotype, the plasmid vector, HBV sequence, cell lines, etc. In our study, we found that both in patient serum and in in vitro studies the sC69* mutant showed lower HBV DNA levels than the WT HBV. In addition, there have been many other truncated HBsAg mutants reported by others showing similar results (Ding et al., 2014; Aragri et al., 2016). Lee et al. (2012) reported that sW182* mutant showed lower HBV DNA levels and existed preferentially in older patients.

There has been little investigation on the influence of HBs mutant on viral infectivity because available and efficient infection cell models are limited. In the past, analyzing how HBsAg mutants influenced HBV infection mostly relied on the hepatitis delta virus (HDV) model, in which HDV hijacks HBV 


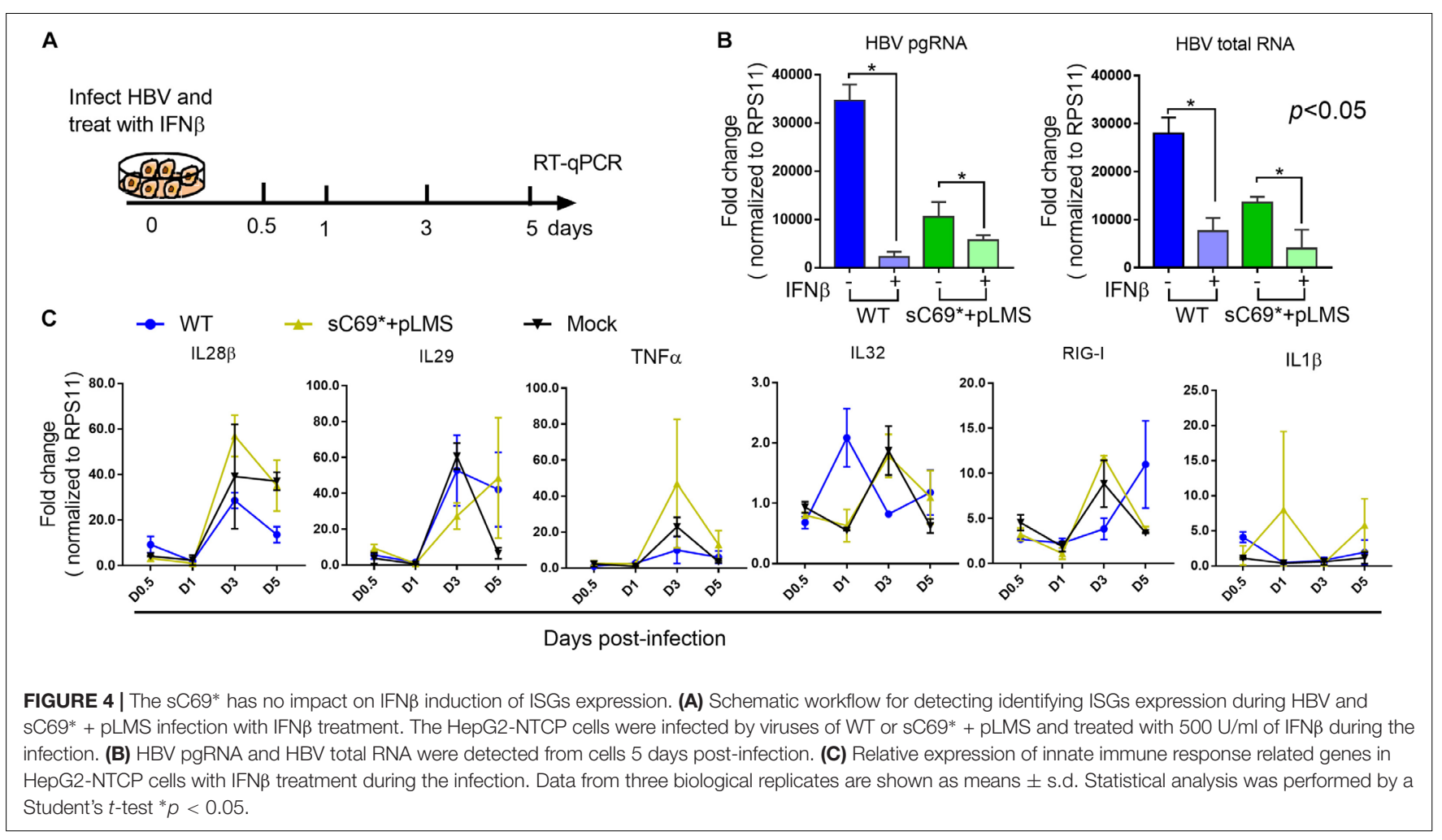

surface proteins for assembly, release and entry into hepatocytes. It has been shown that sI100, sG119, sC124, sK141, sP142, sC147, and $\mathrm{sC} 149$ were critical for both virion formation and viral infectivity (Kwei et al., 2013). Recently, HepG2-NTCP and hESCinduced HLCs were reported to support HBV infection (Yan et al., 2015b; Seitz et al., 2016). We also established effective cell models for HBV infection and used them to study the impact of the sC69* mutant on viral infection and spread. In this study, we tested the impact of the sC69* on viral infectivity and spread in the HepG2-NTCP cell line. In addition, the trans-well system for $\mathrm{HBV}$ infection experiments was shown to be better than applying concentrated viruses directly onto susceptible cells for viral infection.

Interestingly, among 19 patients with the sC69* mutant, 15 patients mutation were found to have a mixture of WT sC69 and sC69* encoded by TGC + TGA. Thus, the questions came out why the sC69* coexisted with WT and how sC69* survived in the host. Based on our data, we proposed the following explanations. The WT HBV rescues the mutant and allows it to complete its life cycle. Warner and Locarnini (2008) showed that sW172* mutant could be rescued by WT for secretion. In addition, Kwei et al. (2013) reported that mutants in the 'a' determinant were also rescued by WT to secrete out of the cells. As shown in our previous report, the sC69* mutant had impaired replication and secretion (Xiang et al., 2017). In this study, we also found that sC69* alone also resulted in deficient virion infection and spread in HepG2-NTCP. The potential mechanism may be that the mutant had truncated HBsAg and negatively affected virion secretion. More studies are needed for further understanding.
In addition, HBV infection in HepG2-NTCP and HLCs models showed an innate immune response during the infection, indicating that these two models can be used to study innate immune responses during HBV infection. However, sC69* could inhibit some immune factors expression (RIG-I, IL29, and ISG15) with or without WT. Moreover, the sC69* mutant could attenuate host innate immune responses to help the WT and itself to escape the host immune surveillance. Thus, the WT and mutant can survive through cooperation relationship in the host. Liu S. et al. (2015) reported that HBsAg could inhibit major vault protein signaling in IFN induction pathways. It is still not clear if $\mathrm{HBsAg}$ mutants impact the host immune response or not. We suspect that the sC69* mutant might show stronger inhibition on the innate immune response than WT. In this study, we used both the HepG2-NTCP cell line and HLCs derived from hESCs as $\mathrm{HBV}$ infection systems to study the $\mathrm{sC69*}$ mutant influence on innate immune responses. The results showed that $\mathrm{sC} 69^{*}$ might have stronger inhibition on the innate immune responses compared to that of the WT HBV. As we know, this should be the first report to relate HBsAg mutant to innate immune responses. When infected HepG2-NTCP cells were treated with poly (I:C), the pgRNA and HBV total RNA levels of sC69* were similar to WT. These indicate that sC69* mutant could persist in the host through escape host innate immune response. They could also use the cooperation strategy to work together to get better viral fitness and persistence. The less innate immune response of sC69* mutant was shown not to be caused by its lower replication, which lead to less virus production. It is more likely that the lower innate immune response is as a result of the surface protein being truncated. However, the issue of how sC69* 


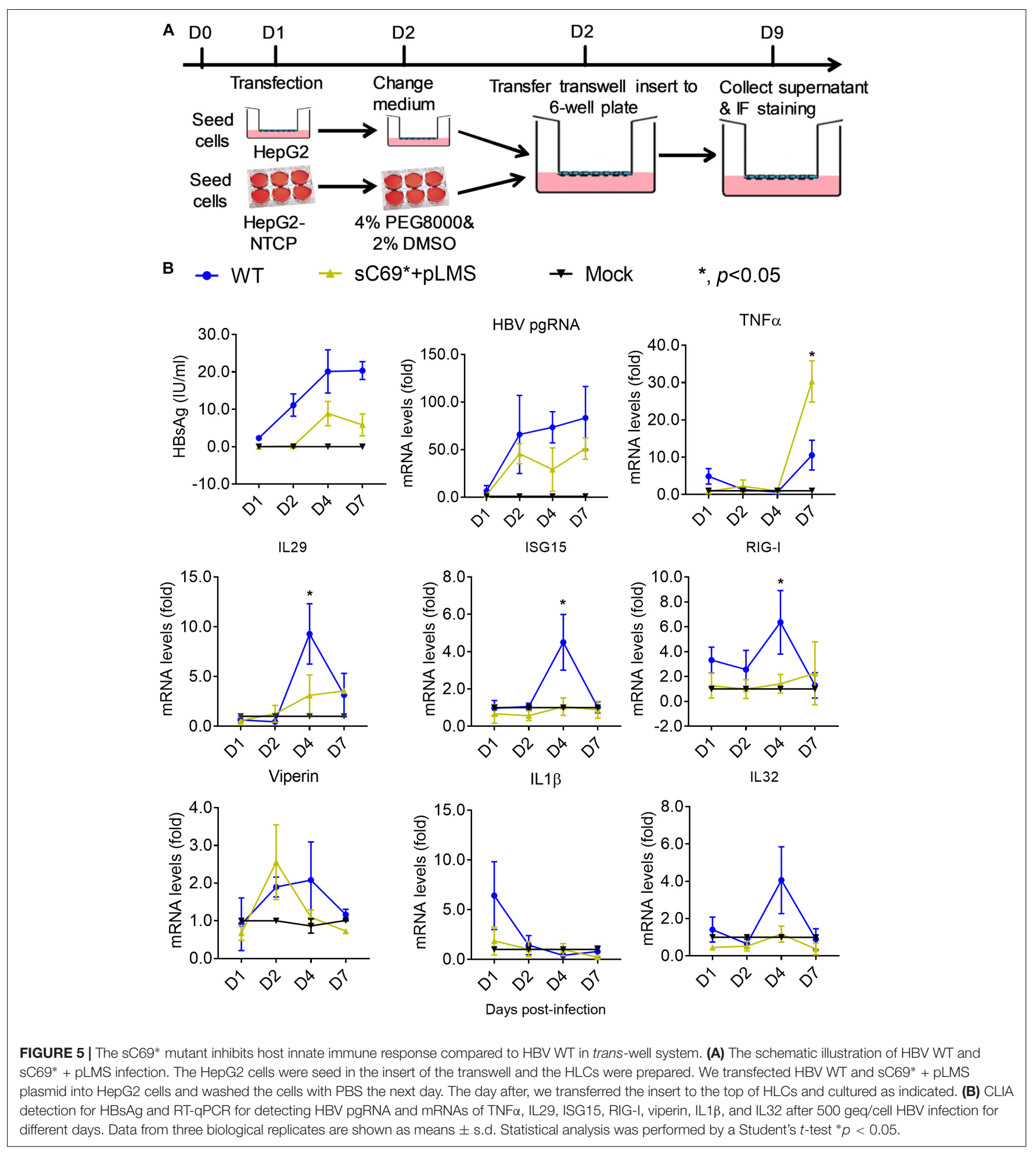

inhibits host innate immune response remains to be elucidated in a future study.

Other publications reported that $\mathrm{HBV}$ did not induce any innate immune response (Cheng et al., 2017; Mutz et al., 2018; Suslov et al., 2018). Our data suggest that WT HBV could slightly induce innate immune response, although we could not detect
ISG-related proteins by Western Blot. Of note, the virus we used is genotype $\mathrm{C}$ rather than genotype $\mathrm{D}$ or $\mathrm{A}$ virus that was used in other studies, which may lead to different IFN responses. Our results are similar to those in other studies (Sato et al., 2015). The sC69*, with the concomitant rtS78T mutation impaired viral replication in vitro, which may explain the association of $\mathrm{sC6}{ }^{*}$ 

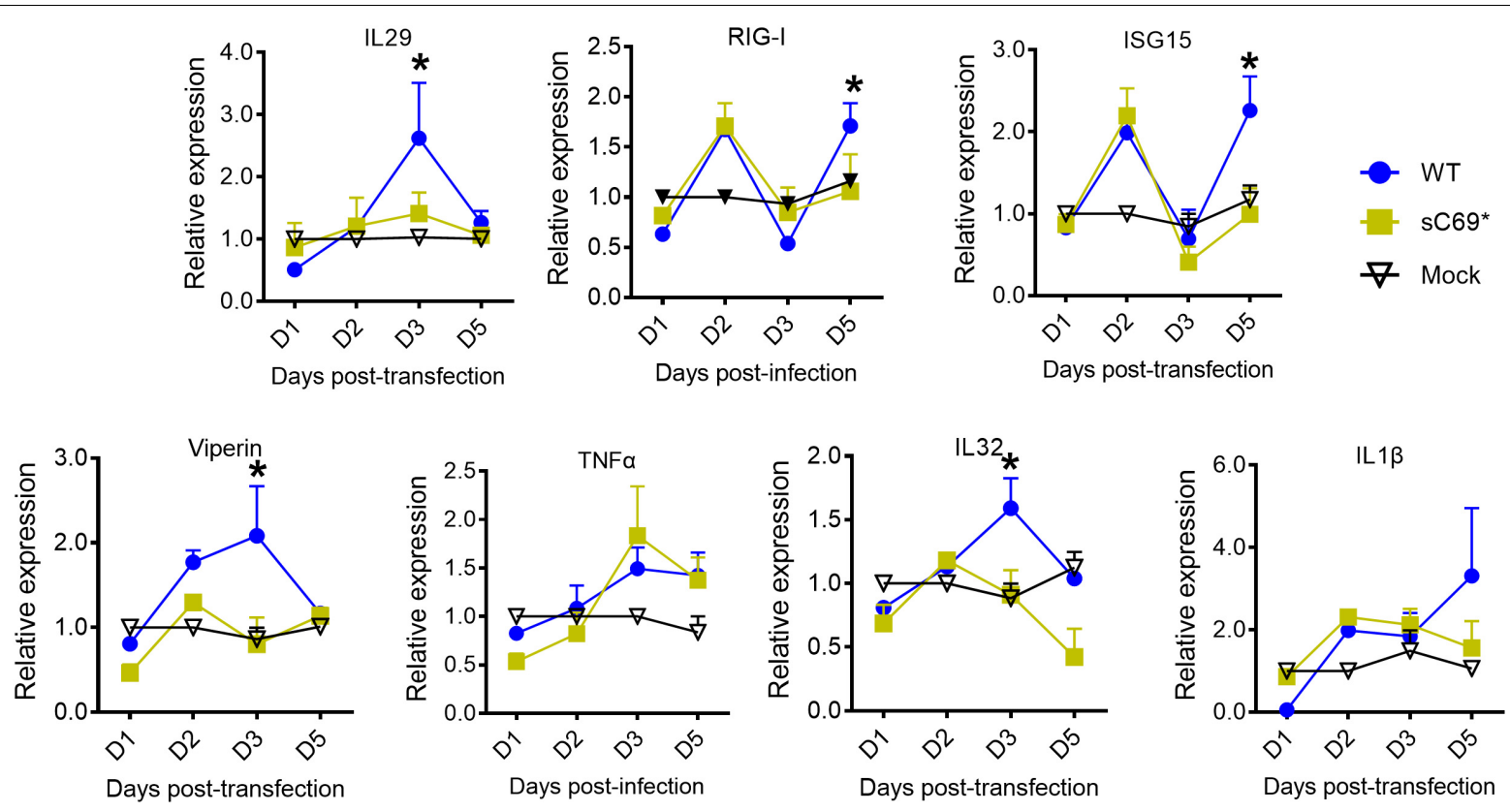

FIGURE 6 | The truncated protein of sC69* attenuates the host innate immune response. RT-qPCR was used to detect the mRNA levels of IL29, ISG15, RIG-I, viperin, IL1 $\beta$, and IL32. Data from three biological replicates are shown as means \pm s.d. Statistical analysis was performed by a Student's $t$-test * $p<0.05$.

with low HBV DNA levels in patient sera. Moreover sC69* was found to inhibit expression of a number of ISGs in vitro and this may possibly lead to attenuation of the innate immune response.

There are some limitations in this study. As mentioned above, some of our results were different from other studies, such as the influence of $\mathrm{sC}^{*} 9^{*}$ on viral replication (ShirvaniDastgerdi et al., 2017). There are perhaps many reasons for this difference, for example the different HBV sequence, plasmid vector, cell lines for HBV replication and infection, genotypes and detection methods (Shirvani-Dastgerdi et al., 2017). In addition, the infection efficiency in HLCs was also low with about $40 \%$ of infected cells, which maybe impact HBV infection and innate immune responses. In addition, how sC69* mutant antagonizes immune response is needed to be studied in the future.

\section{CONCLUSION}

We found that sC69* usually coexists with WT HBV. The sC69* substitution could impact viral infection and be rescued when coexisting with WT. In addition, sC69* could possibly inhibit the innate immune response compared to the $\mathrm{WT}$, allowing the virus to escape immune surveillance.

\section{MATERIALS AND METHODS}

\section{Serum Samples}

Serum samples from $435 \mathrm{CHB}$ patients with genotype C HBV infection were used, which were stored at $-80^{\circ} \mathrm{C}$ from our previous studies (Hao et al., 2015; Liang X. et al., 2015; Su et al., 2016). 369 samples were from treatment-naïve patients
(327 HBeAg-positive and $42 \mathrm{HBeAg-negative).} 66$ samples were from patients with ongoing lamivudine (LMV) treatment. The written informed consents were obtained from the participants of this study. All patients were free for hepatitis $C$ virus, hepatitis $\mathrm{D}$ virus, human immunodeficiency virus, auto-immune liver disease and alcohol/drug abuse. The serological markers for HBV infection were detected as mentioned in our previous study (Xiang et al., 2017).

\section{PCR Amplification, DNA Sequencing and Sequence Analysis}

As described before, we amplified HBV RT sequences covering the entire $S$ gene and sequenced to identify the AA substitutions in SHBs (Ding et al., 2014).

\section{Plasmids}

We cloned the sC69* mutant into plasmid pBB4.5 1.2/PC, which contained a 1.2-fold length $\mathrm{HBV}$ genome of genotype $\mathrm{C}$ with a G1896A mutation in the precore region (Xiang et al., 2017). HBV replication deficient mutation of YMDD to YMHD for WT-YMHD and sC69*-YMHD were cloned based on the WT and sC69* mutant (Xiang et al., 2017). Hemagglutinin (HA) tag was inserted into the $\mathrm{C}$ terminus of surface protein of the WT and after AA68 of sC69* mutant surface protein as described before (Xiang et al., 2017). As shown before, a pBluescript II KS (+) plasmid (Addgene, United States) was used to express HBsAg. In brief, the LHBs coding region was inserted into the KpnI and SacI sites of this vector, which supported expression of SHBs, MHBs, and LHBs and was named as pLMS (Xiang et al., 2017). 


\section{HBV Inoculum Preparation}

Infectious HBV particles were collected from HepDE19 cells (a gift from Haitao Guo, Indiana University) as previously described (Michailidis et al., 2017). Briefly, HepDE19 cells were cultured in tetracycline-free medium to induce HBV virion production. Supernatant was collected every other day and replenished for 20 days. The collected supernatant was concentrated with Centricon ${ }^{\circledR}$ Plus-70 Filter (Millipore, United States) by centrifuging with $2,500 \times g$ for $30 \mathrm{~min}$ at $4^{\circ} \mathrm{C}$. The concentrated $\mathrm{HBV}$ particle stocks were stored at $-80^{\circ} \mathrm{C}$. $\mathrm{HBV}$ inoculum was determined by quantitative PCR (qPCR) of HBV DNA. The quantification of HBV inoculum was expressed as HBV genome equivalent/cell (geq/cell), which was equal to HBV DNA copies. The protocol for HBV DNA quantitation was described before (Shlomai et al., 2014; Xiang et al., 2017).

\section{Differentiation of Human Embryonic Stem Cells (hESCs) Into Induced Hepatic-Like Cells (HLCs)}

The protocol for hESCs (H1) differentiation into HLCs was described before ( $\mathrm{Wu}$ et al., 2018). Confluent hESCs were dissociated into single cells with accutase and seeded into 12-well plates cultured with $\mathrm{mTeSR}^{\mathrm{TM}}$ (knock out DMEM/F-12 with 5\% hESC supplement, StemCell Technologies, Vancouver, Canada). Definitive endoderm (DE) was induced with the Definitive Endoderm Kit (StemCell Technologies, Vancouver, Canada) following the manufacturer's instructions. To differentiate cells to hepatic progenitors, the cells were cultured in the presence of RPMI/B-27 supplemented with $20 \mathrm{ng} / \mathrm{ml} \mathrm{BMP} 4$ and $10 \mathrm{ng} / \mathrm{ml}$ bFGF for 5 days. Then, cells were exposed to RPMI/B-27 with 20 ng/HGF for 5 days. The immature hepatocyte-like cells were cultured in the presence of HCM (Lonza, Switzerland) with $20 \mathrm{ng} / \mathrm{ml}$ oncostatin $\mathrm{M}$ for 5 days with changes of medium every 2 days.

\section{HBV Infection}

HepG2-NTCP and HLCs cells were used for HBV infection. The infection protocol was shown before (Michailidis et al., 2017). The poly (I:C) (Sigma, Shanghai, China), and IFN $\beta$ (PeproTech, Suzhou, China) were used to treat the cells during HBV infection. The details were showed at each experiment.

\section{HBV Infection in Trans-Well System}

Both HepG2-NTCP and HLCs were used for studying the sC69* mutant with WT as a control in vitro. HepG2 cells were seeded to the top and HepG2-NTCP or HLCs were seeded in the bottom of the 24-well trans-well plates, respectively. The HepG2-NTCP cells were treated with $2 \%$ DMSO 1 day before infection. As described before, plasmids were transfected into HepG2 cells in the presence of X-tremeGENE 9 at a ratio of plasmids and transfection reagent $0.5 \mu \mathrm{g}: 1.5 \mu \mathrm{l}$ (Roche Diagnostics, Mannheim, Germany) (Xiang et al., 2017). $12 \mathrm{~h}$ later, the cells were washed five times with phosphate buffered saline (PBS) and then kept in fresh medium. Then, the inserts were transferred into HepG2-NTCP or HLCs trans-well plates and the bottom medium were replaced with fresh medium containing 4\% PEG8000 and cultured for 5 or 7 days (Yan et al., 2015b).

\section{Immunofluorescence Assay}

Cells were fixed with 4\% paraformaldehyde diluted in PBS for 20 min and permeabilized with $0.1 \%$ Triton-X 100 diluted in PBS for $10 \mathrm{~min}$ at room temperature. Then cells were blocked in $10 \%$ goat serum with $1 \%$ bovine serum albumin (BSA) for $60 \mathrm{~min}$ on the shaker, followed by anti-HBc (Austral, United States), anti-HA (Sigma, United States), anti-nanog (Santa Cruz, Dallas, TX, United States), anti-FoxA2 (Sigma-Aldrich, St. Louis, MO, United States), anti-HNF4a (Cell Signaling), anti-AFP (SigmaAldrich, St. Louis, MO, United States) or anti-ALB (Cedarlane, Burlington, Canada) antibodies in 1:500 dilution with $1 \%$ BSA in PBS incubated at $4^{\circ} \mathrm{C}$ over night. The next day, the primary antibody was washed away and the 594 or 488 fluorescent anti-rabbit secondary antibody and $5 \mathrm{mg} / \mathrm{ml} 4^{\prime}, 6-$ DAPI with 1:20,000 dilution were added and incubated at $37^{\circ} \mathrm{C}$ for $30 \mathrm{~min}$. Then, after washing the secondary antibody away, the cells were observed by fluorescent microscopy (Wu X. et al., 2012).

\section{Quantification Analysis of HBV pgRNA and Host Genes}

The quantitative reverse transcription PCR (RT-qPCR) for HBV pregenomic RNA (pgRNA) and host gene expressions were performed as previously described (Shlomai et al., 2014; Xiang et al., 2017). In brief, total HBV RNA was isolated and quantified. First strand cDNA was synthesized using SuperScript III RT kit (Invitrogen). Ribosomal protein S11 (RPS11) gene was used to normalize RT-qPCR results for HBV transcripts. The expression of the host genes tested in this study included IL29, ISG15, RIG-I, IL-1b, IL32, viperin, TNF- $\alpha$, etc., which reflected the host innate immune responses to $\mathrm{HBV}$ infection. The primers were shown in Supplementary Table S1.

\section{Detection of HBsAg and $\mathrm{HBeAg}$}

The HBsAg and HBeAg detection were as previously described (Xiang et al., 2017). In brief, serum HBsAg levels were detected by the Architect HBsAg QT (Abbott) Assay. HBsAg and $\mathrm{HBe}$ Ag levels from cell culture supernatants were detected with commercial CLIA kits (Autobio Diagnostics Co., China).

\section{Statistical Analyses}

Statistical analysis was performed using the SAS version 9.1 software package. Continuous and categorical variables were compared between groups using the student's $t$-test and Chi-square test. Values of $p<0.05$ were considered statistically significant.

\section{ETHICS STATEMENT}

Both the informed and written consents were obtained from the participants of this study. One participant was under the age of 16 , whose written consent was obtained from his parent. 


\section{AUTHOR CONTRIBUTIONS}

TL and HZ created the study concept and design. KX, YX, YL, LH, and LW acquired the data (sample collection, processing, database establishment, etc.). KX and TL analyzed and interpreted the data. KX provided statistical analysis and drafted the manuscript. TL and HZ critically revised the manuscript for important intellectual content.

\section{FUNDING}

This study was supported by financial grants from the Major Science and Technology Special Project of China Thirteenth Five-Year Plan (2017ZX10202202-004-004), the National Natural Science Foundation of China (Grant Nos. 81772174 to TL

\section{REFERENCES}

Alavian, S. M., Carman, W. F., and Jazayeri, S. M. (2013). HBsAg variants: diagnostic-escape and diagnostic dilemma. J. Clin. Virol. 57, 201-208. doi: 10.1016/j.jcv.2012.04.027

Aragri, M., Alteri, C., Battisti, A., Di Carlo, D., Minichini, C., Sagnelli, C., et al. (2016). Multiple hepatitis B virus (HBV) quasispecies and immune-escape mutations are present in HBV surface antigen and reverse transcriptase of patients with acute hepatitis B. J. Infect. Dis. 213, 1897-1905. doi: 10.1093/ infdis/jiw049

Betz-Stablein, B. D., Topfer, A., Littlejohn, M., Yuen, L., Colledge, D., Sozzi, V., et al. (2016). Single-molecule sequencing reveals complex genomic variation of hepatitis B virus during 15 years of chronic infection following liver transplantation. J. Virol. 90, 7171-7183. doi: 10.1128/JVI.00243-16

Borderia, A. V., Isakov, O., Moratorio, G., Henningsson, R., Aguera-Gonzalez, S., Organtini, L., et al. (2015). Group selection and contribution of minority variants during virus adaptation determines virus fitness and phenotype. PLoS Pathog 11:e1004838. doi: 10.1371/journal.ppat.1004838

Cao, L., Wu, C., Shi, H., Gong, Z., Zhang, E., Wang, H., et al. (2014). Coexistence of hepatitis $\mathrm{B}$ virus quasispecies enhances viral replication and the ability to induce host antibody and cellular immune responses. J. Virol. 88, 8656-8666. doi: 10.1128/JVI.01123-14

Chen, J., Wu, M., Wang, F., Zhang, W., Wang, W., Zhang, X., et al. (2015). Hepatitis $B$ virus spliced variants are associated with an impaired response to interferon therapy. Sci. Rep. 5:16459. doi: 10.1038/srep16459

Cheng, X., Xia, Y., Serti, E., Block, P. D., Chung, M., Chayama, K., et al. (2017). Hepatitis B virus evades innate immunity of hepatocytes but activates cytokine production by macrophages. Hepatology 66, 1779-1793. doi: 10.1002/hep. 29348

Ding, H., Liu, B. M., Zhao, C. Y., Yang, J. X., Yan, C. H., Yan, L., et al. (2014). Amino acid similarities and divergences in the small surface proteins of genotype $\mathrm{C}$ hepatitis $\mathrm{B}$ viruses between nucleos(t)ide analogue-naive and lamivudinetreated patients with chronic hepatitis B. Antivir. Res. 102, 29-34. doi: 10.1016/ j.antiviral.2013.11.015

Hao, R., Xiang, K. H., Peng, Y. Q., Hou, J. L., Sun, J., Li, Y., et al. (2015). Naturally occurring deletion/insertion mutations within HBV whole genome sequences in HBeAg-positive chronic hepatitis $\mathrm{B}$ patients are correlated with baseline serum HBsAg and HBeAg levels and might predict a shorter interval to $\mathrm{HBeAg}$ loss and seroconversion during antiviral treatment. Infect. Genet. Evol. 33, 261-268. doi: 10.1016/j.meegid.2015. 05.013

Huang, C. H., Yuan, Q., Chen, P. J., Zhang, Y. L., Chen, C. R., Zheng, Q. B., et al. (2012). Influence of mutations in hepatitis B virus surface protein on viral antigenicity and phenotype in occult HBV strains from blood donors. J. Hepatol. 57, 720-729. doi: 10.1016/j.jhep.2012.05.009

Kwei, K., Tang, X., Lok, A. S., Sureau, C., Garcia, T., Li, J., et al. (2013). Impaired virion secretion by hepatitis $\mathrm{B}$ virus immune escape mutants and its rescue by and 81802002 to $\mathrm{KX}$ ), and the Fund for Fostering Young Scholars of Peking University Health Science Center (Grant No. BMU2017YB001 to KX).

\section{ACKNOWLEDGMENTS}

We thank Prof. Hongkui Deng's Lab for sharing the hES (H1) cells.

\section{SUPPLEMENTARY MATERIAL}

The Supplementary Material for this article can be found online at: https://www.frontiersin.org/articles/10.3389/fmicb. 2019.01341/full\#supplementary-material

wild-type envelope proteins or a second-site mutation. J. Virol. 87, 2352-2357. doi: 10.1128/JVI.02701-12

Lee, S. A., Kim, K., Kim, H., and Kim, B. J. (2012). Nucleotide change of codon 182 in the surface gene of hepatitis B virus genotype $C$ leading to truncated surface protein is associated with progression of liver diseases. J. Hepatol. 56, 63-69. doi: 10.1016/j.jhep.2011.06.028

Liang, T. J., Block, T. M., McMahon, B. J., Ghany, M. G., Urban, S., Guo, J. T., et al. (2015). Present and future therapies of hepatitis B: from discovery to cure. Hepatology. 62, 1893-1908. doi: 10.1002/hep.28025

Liang, X., Cheng, J., Sun, Y., Chen, X., Li, T., Wang, H., et al. (2015). Randomized, three-arm study to optimize lamivudine efficacy in hepatitis B e antigen-positive chronic hepatitis B patients. J. Gastroen. Hepatol. 30, 748-755. doi: 10.1111/jgh. 12835

Liu, S., Peng, N., Xie, J., Hao, Q., Zhang, M., Zhang, Y., et al. (2015). Human hepatitis $\mathrm{B}$ virus surface and e antigens inhibit major vault protein signaling in interferon induction pathways. J. Hepatol. 62, 1015-1023. doi: 10.1016/j.jhep. 2014.11.035

Liu, Y., Li, J., Chen, J., Li, Y., Wang, W., Du, X., et al. (2015). Hepatitis B virus polymerase disrupts K63-linked ubiquitination of STING to block innate cytosolic DNA-sensing pathways. J. Virol. 89, 2287-2300. doi: 10.1128/JVI. 02760-14

Locarnini, S., Bowden, S., and Hepatitis, B. (2012). Surface antigen quantification: not what it seems on the surface. Hepatology 56, 411-414. doi: 10.1002/hep. 25732.

Lucifora, J., Xia, Y., Reisinger, F., Zhang, K., Stadler, D., Cheng, X., et al. (2014). Specific and nonhepatotoxic degradation of nuclear hepatitis B virus cccDNA. Science 343, 1221-1228. doi: 10.1126/science.1243462

Michailidis, E., Pabon, J., Xiang, K., Park, P., Ramanan, V., Hoffmann, H. H., et al. (2017). A robust cell culture system supporting the complete life cycle of hepatitis B virus. Sci. Rep. 7:16616. doi: 10.1038/s41598-017-16882-5

Mutz, P., Mutz, P., Lempp, F. A., Bender, S., Qu, B., Schöneweis, K., et al. (2018). $\mathrm{HBV}$ bypasses the innate immune response and does not protect $\mathrm{HCV}$ from antiviral activity of interferon. Gastroenterology 154, 1791-1804. doi: 10.1053/j. gastro.2018.01.044

Pfeiffer, J. K., and Kirkegaard, K. (2005). Increased fidelity reduces poliovirus fitness and virulence under selective pressure in mice. PLoS Pathog. 1:e11. doi: 10.1371/journal.ppat.0010011

Pollicino, T., Amaddeo, G., Restuccia, A., Raffa, G., Alibrandi, A., Cutroneo, G., et al. (2012). Impact of hepatitis B virus (HBV) preS/S genomic variability on HBV surface antigen and HBV DNA serum levels. Hepatology 56, 434-443. doi: 10.1002/hep. 25592

Rehermann, B., and Nascimbeni, M. (2005). Immunology of hepatitis B virus and hepatitis C virus infection. Nat. Rev. Immunol. 5, 215-229. doi: 10.1038/nri1573

Sato, S., Li, K., Kameyama, T., Hayashi, T., Ishida, Y., Murakami, S., et al. (2015). The RNA sensor RIG-I dually functions as an innate sensor and direct antiviral factor for hepatitis B virus. Immunity 42, 123-132. doi: 10.1016/j.immuni.2014. 12.016 
Seitz, S., Iancu, C., Volz, T., Mier, W., Dandri, M., Urban, S., et al. (2016). A slow maturation process renders hepatitis B virus infectious. Cell Host Microbe 20, 25-35. doi: 10.1016/j.chom.2016.05.013

Shirogane, Y., Watanabe, S., and Yanagi, Y. (2012). Cooperation between different RNA virus genomes produces a new phenotype. Nat. Commun. 3:1235. doi: $10.1038 /$ ncomms 2252

Shirvani-Dastgerdi, E., Winer, B. Y., Celia-Terrassa, A., Kang, Y., Tabernero, D., Yagmur, E., et al. (2017). Selection of the highly replicative and partially multidrug resistant rtS78T polymerase mutation in two patients with chronic hepatitis B virus infection during tenofovir-entecavir combination therapy. J. Hepatol. 67, 246-254. doi: 10.1016/j.jhep.2017.03.027

Shlomai, A., Schwartz, R. E., Ramanan, V., Bhatta, A., de Jong, Y. P., Bhatia, S. N., et al. (2014). Modeling host interactions with hepatitis B virus using primary and induced pluripotent stem cell-derived hepatocellular systems. Proc. Natl. Acad. Sci. U.S.A. 111, 12193-12198. doi: 10.1073/pnas.1412631111

Su, M. Z., Xiang, K. H., Li, Y., Li, Y. T., Deng, J., Xu, X. Z., et al. (2016). Higher detection rates of amino acid substitutions in $\mathrm{HBV}$ reverse transcriptase/surface protein overlapping sequence is correlated with lower serum HBV DNA and HBsAg levels in HBeAg-positive chronic hepatitis B patients with subgenotype B2. Infect. Genet. Evol. 40, 275-281. doi: 10.1016/j.meegid.2016.03.019

Suslov, A., Boldanova, T., Wang, X., Wieland, S., and Heim, M. H. (2018). Hepatitis $\mathrm{B}$ virus does not interfere with innate immune responses in the human liver. Gastroenterology 154, 1778-1790. doi: 10.1053/j.gastro.2018.01.034

Warner, N., and Locarnini, S. (2008). The antiviral drug selected hepatitis B virus rtA181T/sW172* mutant has a dominant negative secretion defect and alters the typical profile of viral rebound. Hepatology 48, 88-98. doi: 10.1002/hep. 22295

Wooddell, C. I., Yuen, M. F., Chan, H. L. Y., Gish, R. G., Locarnini, S. A., Chavez, D., et al. (2017). RNAi-based treatment of chronically infected patients and chimpanzees reveals that integrated hepatitis B virus DNA is a source of HBsAg. Sci. Transl. Med. 9:eaan0241. doi: 10.1126/scitranslmed.aan0241

Wu, C. C., Deng, W. Y., Deng, L., Cao, L., Qin, B., Li, S. X., et al. (2012). Amino acid substitutions at positions 122 and 145 of hepatitis B virus surface antigen (HBsAg) determine the antigenicity and immunogenicity of HBsAg and influence In Vivo HBsAg clearance. J. Virol. 86, 4658-4669. doi: 10.1128/JVI. 06353-11

Wu, X., Robotham, J. M., Lee, E., Dalton, S., Kneteman, N. M., Gilbert, D. M., et al. (2012). Productive hepatitis $C$ virus infection of stem cell-derived hepatocytes reveals a critical transition to viral permissiveness during differentiation. PLoS Pathog. 8:e1002617. doi: 10.1371/journal.ppat.1002617

Wu, X. F., Thi, V. L. D., Huang, Y. M., Billerbeck, E., Saha, D., Hoffmann, H. H., et al. (2018). Intrinsic immunity shapes viral resistance of stem cells. Cell 172, 423-438. doi: 10.1016/j.cell.2017.11.018

Xiang, K. H., Michailidis, E., Ding, H., Peng, Y. Q., Su, M. Z., Li, Y., et al. (2017). Effects of amino acid substitutions in hepatitis B virus surface protein on virion secretion, antigenicity, HBsAg and viral DNA. J. Hepatol. 66, 288-296. doi: 10.1016/j.jhep.2016.09.005

Yan, H., Zhong, G. C., Xu, G. W., He, W. H., Jing, Z. Y., Gao, Z. C., et al. (2012). Sodium taurocholate cotransporting polypeptide is a functional receptor for human hepatitis B and D virus. eLife 1:e00049. doi: 10.7554/eLife. 00049

Yan, R., Zhao, X., Cai, D., Liu, Y., Block, T. M., Guo, J. T., et al. (2015a). The interferon-inducible protein tetherin inhibits hepatitis $\mathrm{B}$ virus virion secretion. J. Virol. 89, 9200-9212. doi: 10.1128/JVI.00933-15

Yan, R., Zhang, Y., Cai, D., Liu, Y., Cuconati, A., and Guo, H. (2015b). Spinoculation enhances HBV infection in NTCP-reconstituted hepatocytes. PLoS One 10:e0129889. doi: 10.1371/journal.pone.0129889

Conflict of Interest Statement: The authors declare that the research was conducted in the absence of any commercial or financial relationships that could be construed as a potential conflict of interest.

Copyright (C) 2019 Xiang, Xiao, Li, He, Wang, Zhuang and Li. This is an open-access article distributed under the terms of the Creative Commons Attribution License (CC BY). The use, distribution or reproduction in other forums is permitted, provided the original author(s) and the copyright owner(s) are credited and that the original publication in this journal is cited, in accordance with accepted academic practice. No use, distribution or reproduction is permitted which does not comply with these terms. 